\title{
Post mortem examinations of HIV infected patients: a nationwide survey
}

\author{
J Davies, I P Everall, P L Lantos
}

\begin{abstract}
Aims-To identify the number and geographical distribution of post mortem examinations performed on HIV infected cadavers; to identify the areas that perform post mortem examinations so that, by invitation and discussion, a national post mortem network could be initiated. Methods-A nationwide survey of Royal College of Pathologists' district tutors (n = 216) was done by postal questionnaire. These were completed by the district tutors or passed on to their histopathologist colleagues. The results were collated after three months. After one follow up letter, an $86 \%$ response rate, which is a significant sample, was achieved.

Results-Twenty eight per cent of respondents were performing HIV post mortem examinations. Of those who were not performing them $(30 \%)$, the lack of clinical or coroner requests was the most common explanation.

All regions provide facilities for HIV post mortem examinations, as judged by the responses.

Conclusions-These findings are of singular importance as it is the first United Kingdom survey investigating the distribution of post mortem examinations on HIV infected cadavers. Studying post mortem material using a systemic, coordinated collection of brain tissue is essential to increase knowledge of a new disease of pandemic proportions.
\end{abstract}

(F Clin Pathol 1993;46:1076-1079)

AIDS was first identified in 1981 in Los Angeles, when unusual illnesses, previously limited to immunocompromised people, developed in apparently healthy young menPneumocystis carinii pneumonia and Kaposi's sarcoma. ${ }^{12}$ Soon, further reports appeared from San Francisco and New York.

Initially, AIDS was characterised by these predominantly systemic illnesses, and the importance of neurological complications was not realised. In 1983 Snider et $a l^{\beta}$ observed that neurological complications frequently developed during the course of the disease. Several large-scale post mortem studies confirmed that the nervous system is affected in over $90 \%$ of patients who died of AIDS ${ }^{4-6}$ and it is now established that the nervous system is a principal target of HIV-1. ${ }^{7}$ In fact, HIV can be isolated from the nervous system a few weeks after infection. ${ }^{8}$ It has also been demonstrated in neural tissues by immunocytochemistry and in situ hybridisation. ${ }^{9}$

Furthermore, it has been realised that there are neuropathological abnormalities which are directly attributed to HIV infection. ${ }^{10}$ These are currently classified as HIV encephalitis, HIV leucoencephalopathy, vacuolar myelopathy and leucoencephalopathy, lymphocytic meningitis, diffuse poliodystrophy and cerebral vasculitis. ${ }^{11}$ Only recently has the effect of HIV on neurons been addressed by quantitative studies which identified clinically important loss in various neocortical areas. ${ }^{12} 13$

Clinically, the term AIDS dementia complex was introduced by Navia et al $(1986)^{14}$ to describe a progressive dementia found in patients with AIDS. Although there is little doubt about the existence of a syndrome of dementia in advanced HIV disease, the term is often confusing in clinical practice, ${ }^{15}$ because it is applied broadly to both minor degrees of impairment and to severe syndromes. Such difficulties were highlighted by staging the AIDS dementia complex ${ }^{16}$ which gave equal importance to both motor and cognitive abnormalities. Thus a patient may be classified as having severe dementia (stage 3) purely on the grounds of severe motor disability. Recently, the American Academy of Neurology AIDS Task Force (1991) ${ }^{17}$ revised the definition, emphasising the importance of cognitive abnormalities and introducing motor and behavioural subcategories as a way of clarifying the degree of impairment. From a neuropathological standpoint, a correlation was suggested between the degree of dementia and the severity of HIV encephalitis, implicating the latter as a cause of the dementia. ${ }^{13} 18$ Neuronal loss, however, may also contribute to dementia as the role of HIV-associated cerebral damage in the development of clinical symptoms is still not fully understood.

Clearly, with our current developing knowledge there is an urgent need to clarify both the neuropathological basis and clinical symptoms of HIV dementia. An understanding of the causes and mechanism of the 
dementia will allow the development of potential treatments to prevent, or even reverse, the cognitive impairments.

Recognising the importance of the neurological complications of HIV infection, the Medical Research Council in 1988 recommended the setting up of a National AIDS Neuropathology Database and Brain Tissue Bank, based at the Department of Neuropathology, Institute of Psychiatry, London. The brain banks consist of a Central AIDS Brain Tissue Bank (London), whose material is obtained predominantly from homosexual men, and two satellite brain banks - one in Oxford, whose material mainly derives from individuals with haemophilia, and the other in Edinburgh with an HIV population of intravenous drug misusers. The aim of the brain banks is to form a systematic coordinated collection of pathologically verified central nervous system tissue from individuals who died with asymptomatic HIV infection or with AIDS. The aim of the accompanying database is to collect pathological and clinical information on all those patients who have undergone post mortem examinations. Thus the brain banks and database provide a resource of information and neural tissue for research groups, including clinicians and scientists.

To date, the database and brain banks mainly receive information and neuropathological material from the specialised centres within the three brain bank areas. As HIV infection is a national problem it was perceived to be particularly important to undertake a survey to estimate the number and distribution of post mortem examinations of HIV infected patients in the United Kingdom. This would firstly, provide information on the national post mortem examination rate and secondly, by identifying centres which perform post mortem examinations, facilitate a national network for collecting material and information. We now present the results of this survey.

\section{Methods}

A nationwide survey of all 216 district tutors, as identified from the published list of the

i) Are post mortems on people who died of HIV disease carried out in your District?

YES

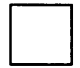

NO

ii) If so, how many of these post mortems were performed during 1990 ?

How many HIV post mortems are likely to be performed during 1991 ?

Format of HIV post mortem examination questionnaire as distributed to the Royal College of Pathologists' district tutors.
Royal College of Pathologists, was undertaken by a postal questionnaire. District tutors, being appointed by pathologists within their district, were considered the most appropriate persons who have access to the information and to the relevant person. Thus the questionnaires were completed by the district tutor or passed on to their histopathologist colleague.

In constructing the postal questionnaire, the aim was to devise a clear, short, and concise form, with no open-ended questions, as these can be difficult to rate. ${ }^{19}$ Consequently, a simple questionnaire, requiring $\mathrm{Yes} / \mathrm{No}$ answers (figure), accompanied by an explanatory letter, was designed to ascertain: (a) the geographical distribution of HIV post mortem examinations in the United Kingdom, and (b) the number of post mortem examinations undertaken in 1990 and the first nine months of 1991 .

Initially, $68 \%$ of the questionnaires were returned, but after a follow up letter this rose to $86 \%$ and so provided a significant sample for the study. ${ }^{20}$

\section{Results}

The results were collated following a three month response period. The results of the study include all post mortem examinations for 1990 and those up to September 1991 . The questionnaires were analysed firstly, by responses, and secondly, according to health region.

\section{RESPONSE}

The replies were initially divided into three main groups and the summary of the results is provided in the table.

The first group were those respondents who were performing HIV post mortem examinations. This group totalled $28 \%$ of the overall response figure. This group was subdivided into four subgroups: $34 \%$ of those willing to perform post mortem examinations were doing so in both 1990 and $1991 ; 41 \%$ had facilities for HIV post mortem examinations but none had taken place; $14 \%$, had performed post mortem examinations in 1990 but had not yet started in 1991; and $11 \%$ of respondents had begun post mortem examinations only in 1991.

The second group comprised $30 \%$ of respondents who were not performing post mortem examinations and who provided explanations for this. The reasons included

\begin{tabular}{ll} 
Table of responses from survey \\
\hline Response & Reason given \\
\hline Yes $(28 \%)$ & Both 1990 and $1991(34 \%)$ \\
& Neither 1990 nor $1991(41 \%)$ \\
& 1990 but not $1991(14 \%)$ \\
No $(30 \%)$ & No but not $1990(11 \%)$ \\
& No facilities $(68 \%)$ \\
& Only in exceptional circumstances $(12 \%)$ \\
No $(28 \%)$ & No desire to $(12 \%)$
\end{tabular}

Figures in parentheses are frequencies of reason given for each group. 
no requests from clinicians or coroners to do post mortem examinations (68\%), lack of suitable facilities to perform high risk post mortem examinations ( $8 \%)$, that post mortem examinations would be done only in exceptional circumstances (12\%), and lastly, the wish not to do them at all (12\%). The third group (28\%) were not performing post mortem examinations and provided no further information.

\section{HEALTH REGIONS}

The replies were also analysed geographically according to health regions. Within the regions the various health districts were identified which were performing HIV post mortem examinations during 1990 and up to September 1991.

The results show that all regions provide facilities for HIV post mortem examinations. The overall number of health districts performing post mortem examinations is quite similar in each region. This must not be confused with the actual number of post mortem examinations undertaken in the various districts which may differ greatly throughout the United Kingdom. While some health districts indicated that the post mortem examination figure was often in single figures, in the four London health regions the number may exceed 100.

\section{Discussion}

These findings are of singular importance as it is the first survey in the United Kingdom investigating the distribution of post mortem examinations of $\mathrm{HIV}$ infected individuals. From the results it is clear that even though only $28 \%$ of health districts perform HIV post mortem examinations, the geographical distribution is such that all health regions have the facilities for them.

The importance of obtaining information in the distribution of post mortem examinations is apparent when considering the applicability of such a procedure. Post mortem examinations are particularly important in diagnosing diseases that could not otherwise have been confirmed during the patient's life. Throughout the development of medical science, the post mortem examination has been important for the recognition and description of new diseases, for the clarification of pathogenesis, and for the evaluation of the effectiveness of new treatment. ${ }^{21}$ It has also assisted in providing knowledge for improving accuracy in clinical diagnoses and treatment methods, thus refining the concept of most disease entities. ${ }^{21}$ Therefore it can be considered that "the autopsy introduced the modern era of enlightenment in medicine, and for 100 years it was the most important instrument in the investigation of disease and its clinical manifestations". ${ }^{22}$

However, with the recent advances in medical technology, such as neuro-imaging, there has been a decline in the perceived value of the additional information gained from post mortem examinations and subsequently a decrease in the post mortem examination rate. ${ }^{23}$ The declining interest in post mortem examinations by clinicians can be explained for the following reasons. Firstly, from a clinical viewpoint the post mortem examination is not regarded as an essential part in the treatment of a patient and is now often seen as providing little new information to that already available from various clinical tests or learned during surgery. Requesting a post mortem examination from a bereaved family can also be a painful task. Furthermore, it can delay funeral arrangements and is time consuming. ${ }^{22}$. Often it is forgotten that new knowledge even today is frequently derived from post mortem examinations, particularly from studies of a number of patients with the same condition. ${ }^{24}$ AIDS is the most recent reminder of this fact.

In an ever progressing field the post mortem needs to be restored to its former position by acknowledging its potential role in clinical practice, teaching, and research. It is a focal point for integrating all sources of knowledge bearing on disease; a "stable chemical end-point" from which to integrate the reasoning back to the beginnings of illness". ${ }^{22}$ The post mortem examination can help to bridge the widening gap between rapid advancements in science and offer an opportune means of seeing the patient in full perspective.

Reinstatement of the post mortem examination to its former role, however, may require the need to involve its incorporation into the contemporary medical team and practices. "This patient, exhaustively studied by a multitude of individuals and by the most modern methods, may die and is then subjected to an autopsy routine which has changed little in a half century or more". ${ }^{25}$ The optimum of medical care, "of which the autopsy represents the final step, can be thought of as a continuum comprising all the patient-doctor encounters during an individual's care and treatment. Primarily directed toward the well-being of the patients, the continuum of medical care has a secondary and time-honoured objective of developing new knowledge, of increasing our information, so that future patients are more effectively treated". ${ }^{25}$

Thus there may be a need to expand the multidisciplinary care team of specialists to include active participation of pathologists. The pathologist would then be able to encourage collaboration and cooperation with colleagues and to explain and educate the specialty in a way that is meaningful and useful to them. Education needs to be addressed by the medical profession and society as a whole to make both more aware of discoveries gained from the post mortem examination in the past and its importance as a contributor to medical knowledge now and in the future. As emphasised by Goodale (1978), ${ }^{26}$ "the public deserves to be better educated about health and disease, about medical procedures in general and about autopsies in particular. What better way to educate them 
than by explaining the findings of post mortem examinations to family members?".

A recent report of the Joint Working Party of the Royal College of Pathologists, Physicians of London, and Surgeons of England, prompted by a decline in the necropsy rate, provided an overwhelmingly strong case for post mortem examinations in medical examination and practice. It also provides practical guidelines and makes recommendations for the use of them in clinical audits. ${ }^{27}$

These issues are particularly relevant to AIDS, a relatively new disease with complicated and complex symptomatology usually caused by more than one pathology. A review of necropsy findings in 101 adult patients with AIDS in two New York hospitals showed that $75(74 \%)$ of the patients had AIDS-related pathology at post mortem examination which had been undiagnosed clinically.$^{28}$ This high rate of unsuspected disease is the strongest case for necropsies in patients with AIDS.

Such issues regarding the post mortem examination are especially pertinent to AIDS. During the past decade an enormous amount of knowledge has accumulated regarding $\mathrm{HIV}$, including the mechanism of infection and the diseases associated with it. Studying post mortem examination material using a systematic, coordinated collection of brain tissue is essential not only for further understanding of the disease process, but also for devising therapeutic regimens and methods of prevention. Without a systematic study of large, well characterised collections of neural material we would not have had the insight into the cellular and molecular mechanisms of HIV infection in the nervous system.

We cannot ignore the vital work being done with post mortem examination material, donated by those with HIV or AIDS and their relatives to further the understanding and progress to more effective treatments of this disease. Our knowledge and understanding of HIV associated dementia and neuronal loss could not have been achieved without this valuable source.

The authors wish to thank the Royal College of Pathologists for providing the list and all those colleagues who collaborated and returned the questionnaires. JD and IE are supported by the Medical Research Council.

1 Centers for Disease Control. Pneumocystis pneumoniaLos Angeles. Morbid Mortal Weekly Rep 1981;30:250-2.

2 Centers for Disease Control. Kaposi's sarcoma and pneumocystis pneumonia among homosexual men-New
York City and California. Morbid Mortal Weekly Rep 1981;30:305-8.

3 Snider WD, Simpson DM, Nielsen S, Gold JWM Metroka CE, Posner JB. Neurological complications of acquired immune deficiency syndrome: Analysis of 50 patients. Ann Neurol 1983;14:403-18.

4 Petito CK, Cho ES, Lemann W, Navia BA, Price RW. Neuropathology of acquired immunodeficiency syndrome (AIDS): An autopsy review. $\mathcal{F}$ Neuropathol Exp Neurol 1986;45:635-46.

5 Budka H, Costanzi G, Cristina S, et al. Brain pathology induced by infection with human immunodeficiency virus (HIV). A histological, immunocytochemical, and electron microscopical study of 100 autopsy cases. Acta Neuropathol 1987;75:185-98.

6 Lantos PL, McLaughlin JE, Scholtz CL, Berry CL, Tighe JR. Neuropathology of the brain in HIV infection. Lancet 1989;863:309-11.

7 Everall IP, Lantos PL. The neuropathology of HIV: a review of the first 10 years. Int Rev Psychol 1991;3 307-20.

8 Ho DD, Rota TR, Schooley RT, et al. Isolation of HTLVIII from cerebrospinal fluid and neural tissues of patients with neurologic syndromes related to the acquired immunodeficiency syndrome. $N$ Engl $7 \mathrm{Med}$ 1985;313:1493-7.

9 Wiley CA, Schrier RD, Nelson JA, Lampert PW, Oldstone MBA. Cellular localization of human immunodeficiency virus infection within the brains of immunodeficiency virus infection within the brains of acquired immune deficiency syndro

10 Sharer LR, Cho ES, Epstein LG. Multinucleated giant cells and HTLV-III in AIDS encephalopathy. Hum Pathol 1985;16:760.

11 Budka $\mathrm{H}$, Wiley CA, Kleihues $\mathrm{P}$, et al. HIV-associated disease of the nervous system: review of nomenclature and proposal for neuropathology-based terminology. Brain Pathol 1991;1:143-52.

12 Everall IP, Luthert PJ, Lantos PL. Neuronal loss in the frontal cortex in HIV infection. Lancet 1991;337: 1119-21.

13 Wiley CA, Masliah E, Morey M, et al. Neocortical damage during HIV infection. Ann Neurol 1991;29:651-7.

14 Navia BA, Jordan BD, Price RW. The AIDS dementia complex: I. Clinical Features. Ann Neurol 1986;19: 517-24.

15 Catalan J. Psychiatric manifestations of HIV disease. Bailliere's Clin Gastroenterol 1990;4:547-62.

16 Price RW, Sidtis JJ, Brew BJ. AIDS dementia complex and HIV-1 infection: a view from the clinic. Brain Pathol 1991;1:155-62.

17 Report of a Working Group of the American Academy of Neurology AIDS Task Force. Nomenclature and Neurology AIDS Task Force. Nomenclature and research case definitions for neurologic manifestations
of human immunodeficiency virus-type 1 (HIV-1) infecof human immunodeficiency

18 Price RW, Brew B, Sidtis J, Rosenblum M, Scheck AC, Cleary $P$. The brain in AIDS: central nervous system HIV-1 infection and AIDS dementia complex. Science 1988;239:586-92.

19 Sudman S, Bradburn NM. Asking questions. In: $A$ practical guide to questionnaire design. London: Jossey-Bass, 1983:262.

20 McCall RB. Fundamental statistics for behavioural sciences. 5 th edn. London: Harcourt Brace Jovanovich, 1990.

21 Hazard JB. The autopsy. ҰAMA 1965;193:149-50.

22 Angrist $\mathrm{A}$. What remedies for the ailing autopsy? $f A M A$ 1965;193:150-2.

23 Goldman L, Sayson R, Robbins S, Cohn LH, Bettmann $M$, Weisberg $M$. The value of the autopsy in three medical eras. N Engl f Med 1983;308:1000-5.

24 Roberts WC. The autopsy: its decline and a suggestion for its revival. N Engl F Med 1978;299:332-8.

25 McManus JFA. The autopsy as research. fAMA 1965; 193:152-4.

26 Goodale F. The future of the autopsy. Am $\mathcal{f}$ Clin Pathol 1978;69:260-2

27 Report of the Joint Working Party of the Royal College of Pathologists, the Royal College of Physicians of Pathologists, the Royal College of Physicians of The Autopsy and audit. London: The Royal College of The Autopsy and aud

28 Wilkes MS, Fortin AH, Felix JC, Godwin TA, Thompson WG. Value of necropsy in acquired immunodeficiency syndrome. Lancet 1988;860:85-8. 